\title{
Involvement of oviduct in sperm capacitation and oocyte development in pigs
}

\author{
H. Rodriguez-Martinez ${ }^{1}$, P. Tienthai ${ }^{1,2}$, K. Suzuki ${ }^{3}$, H. Funahashi ${ }^{4}$, \\ H. Ekwall ${ }^{2}$ and A. Johannisson ${ }^{2}$
}

Departments of 'Obstetrics and Gynaecology and ${ }^{2}$ Anatomy and Histology, Faculty of Veterinary Medicine, Swedish University of Agricultural Sciences (SLU), PO Box 7039, SE-750 O7 Uppsala, Sweden; ${ }^{3}$ Experimental Farm, Faculty of Agriculture, Hokkaido University, Sapporo 060-0811, Japan; and ${ }^{4}$ Laboratory of Animal Reproduction, Faculty of Agriculture, Okayama University, Okayama 700-8530, Japan

An overview is presented on the structure and function of the pig oviduct in relation to sperm capacitation and oocyte development in vivo. In pigs, a functional sperm reservoir is established in the uterotubal junction-isthmus when sperm deposition occurs before ovulation. Capacitation is assumed to occur in this location, and spermatozoa progress towards the ampullaryisthmic junction at about the time of ovulation as a consequence of capacitation and hyperactivation. Preliminary data from our laboratory on viable spermatozoa retrieved from the sperm reservoir and the ampullaryisthmic junction of mated sows at pre- and periovulation oestrus showed that the largest subpopulation (60-90\%) was of uncapacitated spermatozoa (using merocyanine-540), whereas $6-37 \%$ of the gated cells were capacitated spermatozoa. Incubation in a capacitation-inducing medium (bicarbonate-containing modified Brackett-Oliphant medium; $\mathrm{mBO}$ ) for $<30$ min effected capacitation readily, more markedly in ampullary-isthmic junction samples than in samples from the uterotubal junction, thereby indicating that uncapacitated spermatozoa responded to the addition of the effector bicarbonate at concentrations similar to those recorded in the periovulatory ampullary-isthmic junction in vivo. Addition of preovulatory isthmic oviductal fluid and hyaluronan under a similar incubation regimen maintained tubal sperm viability without obvious induction of capacitation. This finding indicates that, before ovulation, the intraluminal fluid of the sperm reservoir might delay sperm capacitation, perhaps because of its hyaluronan content. Evidence is presented that the sperm population in the oviduct undergoes capacitation under particular conditions in the upper tubal compartments. The diverse response of spermatozoa to capacitation stimuli helps to ensure full rates of fertilization in vivo. Data are also provided on the importance of final zona pellucida maturation in the pig oviduct to warrant proper zona pellucida reaction after sperm penetration, which would address in part the abnormal occurrence of polyspermy in in vitro fertilization of pigs. 


\section{Introduction}

Despite its fairly simple appearance, the pig oviduct is where essential reproductive processes occur, including the preparation of gametes for fertilization, fertilization and the initial phases of embryonic development. With the rapid development of in vitro assisted reproductive techniques, these processes can be performed in the laboratory with a reasonable degree of success. Spermatozoa can be capacitated, and oocytes from offal can be matured and fertilized. Further culture of the resulting zygotes allows production of early embryos that can be transferred, establish pregnancies and produce live offspring. These in vitro techniques, albeit suboptimal, have provided a large amount of valuable information on sperm capacitation, sperm-oocyte binding, oocyte maturation, fertilization and the early cleavage stages of newly developing embryos. However, failure of fertilization due to incomplete oocyte maturation and use of unphysiologically high numbers of capacitated spermatozoa can lead to lethal polyspermy and impaired developmental capability, and are major constraints during in vitro fertilization (IVF) in pigs. Owing to the conditions under which gamete preparation for fertilization, fertilization itself and early embryo development differ in vitro and in vivo (see Rodriguez-Martinez et al., 1998), there is a need to examine the way in which the oviductal structure and function carry out these tasks. This is the intention of this review, which focuses on the formation of the tubal sperm reservoir, the occurrence of capacitation in tubal spermatozoa and some characteristics of prefertilization oocytes, particularly the zona pellucida.

\section{General features of pig oviducts}

The oviduct of a mature pig is approximately $25 \mathrm{~cm}$ in length and has a simple tubular histology, with an endosalpinx, a double-layered myosalpinx and a serosal mesosalpinx. The oviduct is divided into three main segments: the narrow isthmus (connected to the uterine horn via the uterotubal junction), the wider ampulla and the even broader, thin-walled infundibulum. The isthmus and ampulla are connected by an inconspicuous ampullaryisthmic junction, whereas the infundibulum is connected to the adovarian fimbriae and ovarian bursa by the abdominal opening (ostium). The endosalpinx is the inner non-glandular mucosa, the plicated lamina propria (Fig. $1 \mathrm{a}-\mathrm{f}$ ) of which is covered with a lining epithelium with ciliated (most cells) and non-ciliated (secretory) cells (Fig. 2b-f). Along this organ, towards the ostium, the longitudinal mucosal plicae acquire secondary and tertiary plicae, making the lumen more complex (Fig. 1c-f). The number of ciliated cells increases while the thickness of the internal, circular smooth muscle becomes thinner. The anatomical segments are considered to be particular tubal compartments that relate to specific functions such as the sperm reservoir (the uterotubal junction-adjacent isthmus), the site of fertilization (the ampullary-isthmic junction) and the area where ovum pick-up is performed (the fimbriae).

\section{The tubal fluid}

The intraluminal oviductal fluid provides the environment in which gametes are transported and matured, and where fertilization and early embryonic development occur. The tubal fluid is formed by specific oviductal secretions and by the passage of fluids and ions (Nichol et al., 1992; Leese et al., 2001). Tubal fluid differs from blood plasma in ionic composition (it is rich in $\mathrm{K}^{+}$and $\left.\mathrm{HCO}_{3}^{-}\right), \mathrm{pH}$, osmolarity and macromolecular content, which vary with the hormonal environment (Nichol et al., 1998). In the pig ampulla, concentrations of 33-35 $\mathrm{mmol} \mathrm{HCO}_{3}{ }^{-}$ $\mathrm{I}^{-1}$ can be recorded during periovulation ( $\mathrm{E}$. Ekstedt and $\mathrm{H}$. Rodriguez-Martinez, unpublished). 

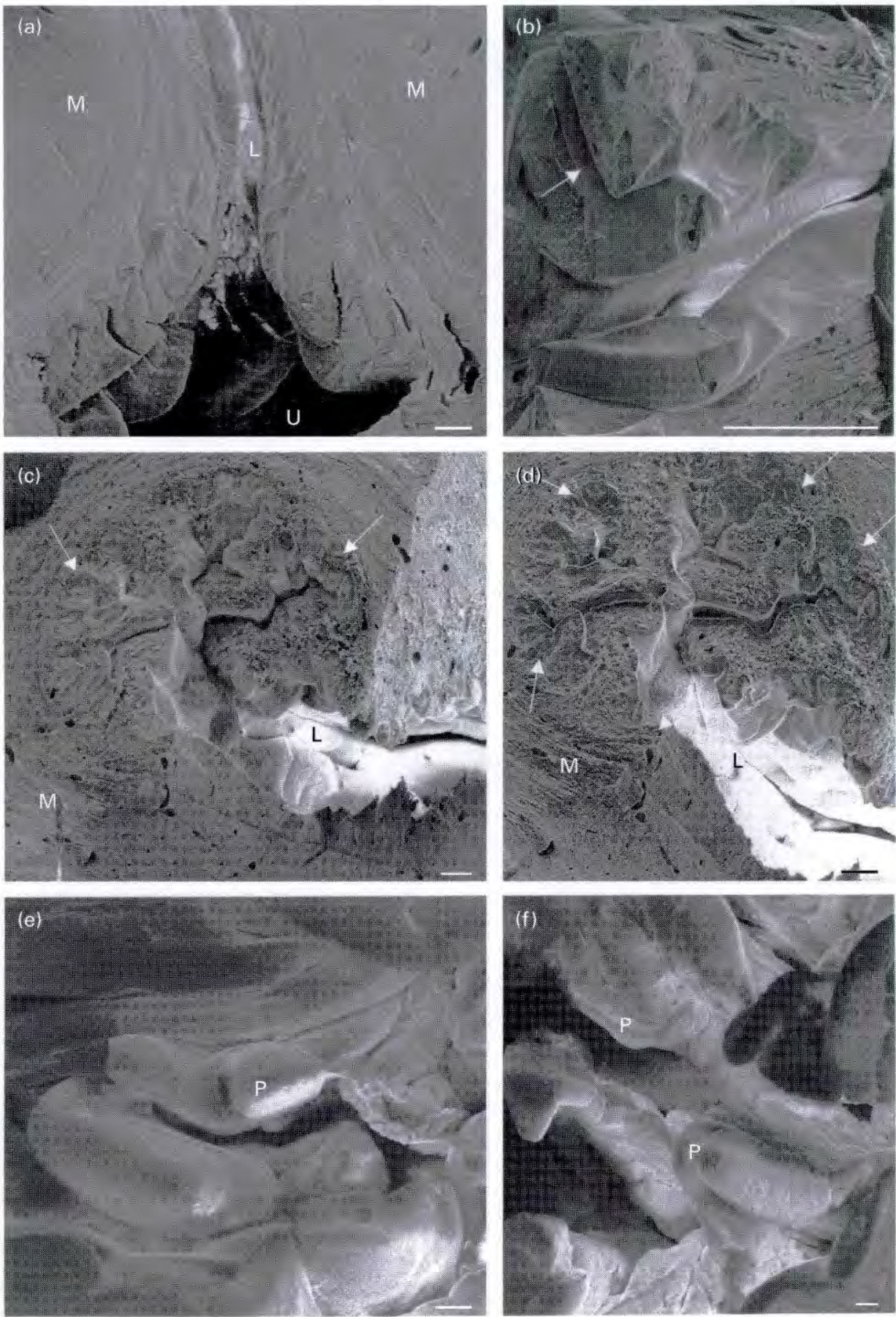

Fig. 1. Scanning electron micrographs of the main segments of the pig oviduct, with special reference to the tubal lumen. (a) A longitudinal section of the uterotubal junction. U: uterus; L: lumen; M: smooth muscle. (b) Open lumen of the uterotubal junction (arrow indicates deep folds), which together with the (c) adjacent isthmus (arrows indicate the deep furrows of the reservoir) builds up the periovulatory sperm reservoir. (d) Upper isthmus has secondary mucosal folds (arrows indicate deep furrows). (e) Lumen of the ampullary-isthmic junction and ( $\mathrm{f}$ ) ampulla with mucosal tertiary plicae (P). Scale bars represent (a) $5 \mathrm{~mm}$, (b) $1 \mathrm{~mm}$ and (c-f) $100 \mu \mathrm{m}$. 

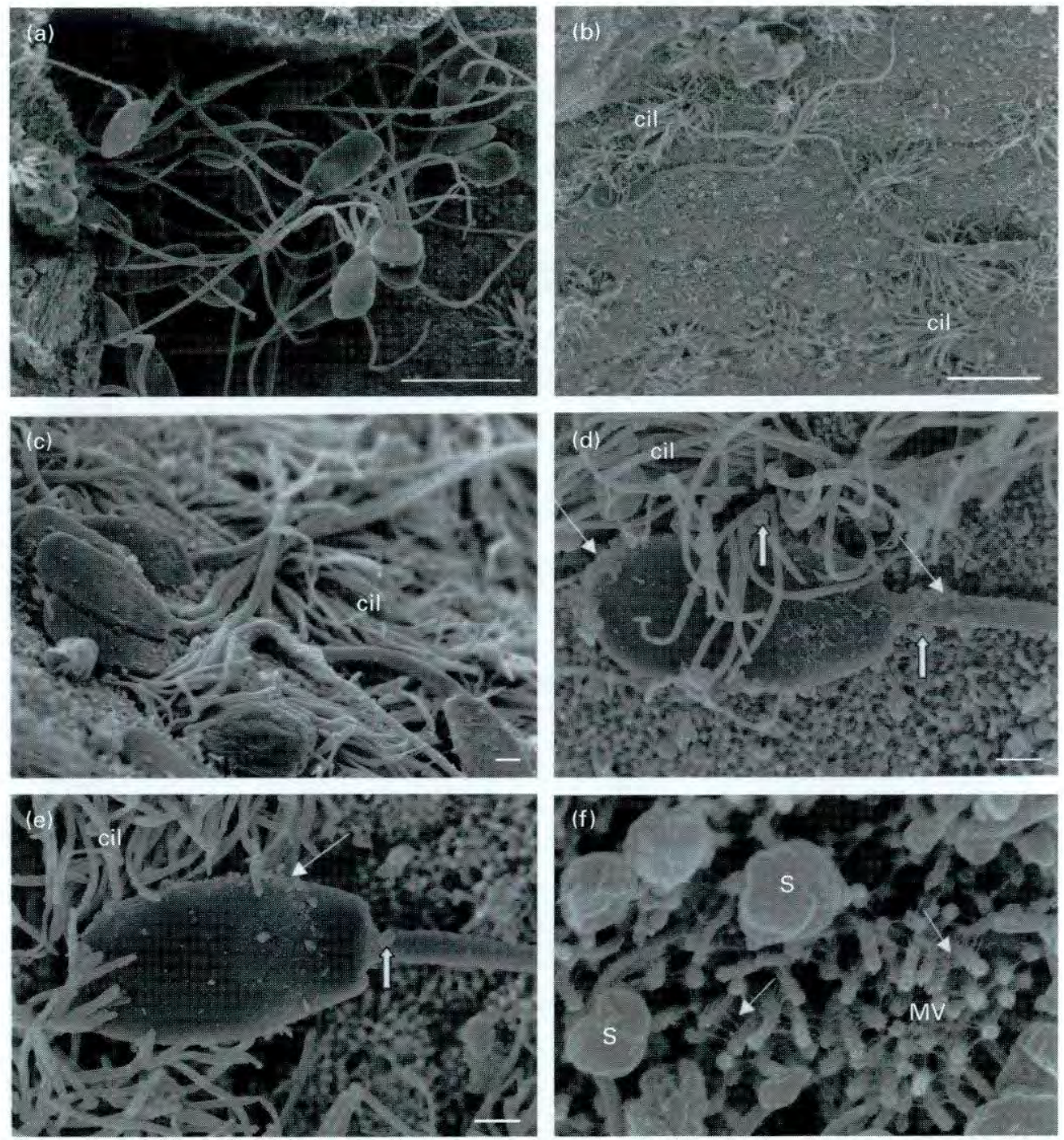

Fig. 2. Scanning electron micrographs of the pig sperm reservoir during preovulatory oestrus. (a) Clusters of spermatozoa in a deep furrow. (b) Spermatozoa apposed to the lining epithelium, with their heads towards the ciliated cells (cil). (c-e) Higher magnifications of spermatozoa in the reservoir depicting the relationship between ciliated cells and the sperm heads. Note the associated secretory droplets (thick arrows) and particulated material (thin arrows). (f) High magnification of the apical surface of secretory cells showing secretory droplets (S) and filament-like intraluminal material (arrows) between the microvilli (MV). Scale bars represent $(a, b) 10 \mu \mathrm{m}$ and (c-f) $1 \mu \mathrm{m}$.

By sampling of isthmic and ampullar segments in vivo using $1.2 \mathrm{~mm}$ indwelling chronic polyethylene catheters, ensheathed in $2.2 \mathrm{~mm}$ outer diameter Silastic tubing, placed surgically in the isthmus and ampulla with a partially modified technique after Kavanaugh et al. (1992), we recorded that fluid volume is highest during oestrus, and that the ampulla produces twice the volume that the isthmus does (Fig. 3), thereby reflecting its greater surface area (P. Tienthai and H. Rodriguez-Martinez, unpublished). The protein content of the oviductal fluid is about $10-15 \%$ of that in serum and originates from selective transudation (mostly at the ampulla) 


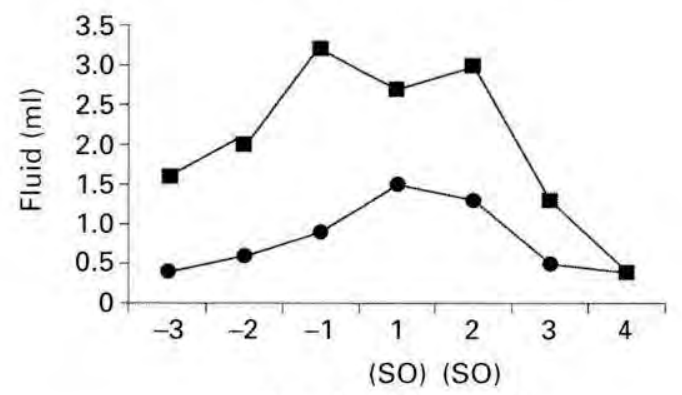

Day of oestrous cycle

Fig. 3. Relative mean daily volumes of pig isthmic (-) and ampullar fluid collected via indwelling catheters between pro-oestrus and metoestrus ( $n=4$ animals). SO: standing oestrus.

and, to a lesser extent, from the lining epithelium. Under oestrogen stimulation, especially during the periovulatory period, the lining epithelium produces specific glycoproteins (Hunter, 1994a; Buhi et al., 1997) such as the oestrous-associated oviduct-specific glycoprotein (OSGP) (Leese et al., 2001). During preovulation, mucopolysaccharides are also formed, particularly at the isthmic segment, where they obliterate the characteristically narrow lumen in which spermatozoa eventually reside (Johansson et al., 2000; Rodriguez-Martinez, 2000). Glycosaminoglycans (GAGs) are also present in the tubal fluid, with a tendency to reach maximum concentrations during oestrus (Tienthai et al., 2000). Heparan sulphate and the non-sulphated hyaluronan have been localized in the epithelium of the pig preovulatory reservoir, often near sperm clusters. Recent immunohistochemical investigations in our laboratory have indicated that the hyaluronan receptor CD44 and the enzymes hyaluronan synthase 3 and hyaluronidase 2 are localized in the lining epithelium of particular regions of the pig oviduct (P. Tienthai, A-S. Berqvist and H. Rodriguez-Martinez, unpublished). Hyaluronan has been related to the modulation of sperm capacitation (Suzuki et al., 2000), the intratubal masking of spermatozoa, as a facilitator of sperm progression towards the site of fertilization and also as an attractant for spermatozoa during fertilization, although much evidence is yet circumstantial (see Rodriguez-Martinez, 2000). Mucopolysaccharides, GAGs and glycoproteins may have a function that is unique to the in vivo condition, increasing viscosity in the luminal fluid, thereby stabilizing the milieu surrounding the gametes and embryos in terms of fluid loss, osmotic changes, relationship to the epithelium and immunological protection.

\section{The functional sperm reservoir}

After deposition in the cervix, spermatozoa are transported rapidly through the uterus and colonize the first segment of the oviduct; $1-2 \times 10^{8}$ spermatozoa enter the uterotubal junction and the adjacent first isthmic segment within 15-20 min (see Hunter, 1990). Here, they form a substantial functional sperm reservoir in which most spermatozoa maintain normal ultrastructure and viability (Rodriguez-Martinez et al., 1990; Mburu et al., 1997). A certain number of these spermatozoa, usually several thousand, are released sequentially from the reservoir towards the site of fertilization at the ampullary-isthmic junction (Hunter, 1990; Mburu et al., 1997). Of these, only a few spermatozoa reach the site of fertilization 
(Hunter, 1995; Mburu et al., 1997) and it is this marked decrease in sperm numbers along the tube that largely prevents polyspermy during fertilization. Most spermatozoa are localized in the narrow deep furrows in the reservoir (Figs 1c and 2a) and, thus, it seems that they make contact with the lining epithelium by their rostral region, as observed in oviducts fixed and processed conventionally for scanning electron microscopy (SEM) (Fig. 2b-e). However, when oviducts are cryofixed and observed under a cryo-SEM, spermatozoa are observed immersed in an amorphous intraluminal material (tubal fluid), rather than in direct contact with the epithelium (Rodriguez-Martinez et al., 1998). Remnants of such secretory material can sometimes be seen among microvilli in samples fixed and processed conventionally (Fig. 2f).

The main function of the sperm reservoir is to ensure that at the time of fertilization adequate, although not excessive, numbers of fertile spermatozoa are present in the midoviduct. To achieve this goal the sperm reservoir entraps large numbers of spermatozoa in a restricted segment, the uterotubal junction-lower isthmus, which during the preovulatory period has a narrow lumen, an oedematous lamina propria, and is filled with a viscous tubal fluid. In addition, the reservoir maintains sperm viability and fertilizing capacity, escaping rejection by the female immune system and, presumably, modulates capacitation before ovulation. Finally, the reservoir prevents polyspermy by retaining spermatozoa and controlling the release of spermatozoa towards the site of fertilization, either continuously or at a given, as yet unknown, signal.

The preovulatory sperm reservoir is built through the concerted action of several factors (see Hunter, 1990; Rodriguez-Martinez et al., 1998), including purely mechanical (for example, the first segment encountered, deep folds, narrow lumen, viscous intraluminal fluid) and active interactions with this microenvironment. These interactions may inhibit sperm metabolism and, hence, motility, and stabilize the plasmalemma by specific signalling. Such interactions may occur with the surrounding fluid and include a decreased temperature or decreased $\mathrm{pH}$ and bicarbonate concentrations (Rodriguez-Martinez et al., 1998; E. Ekstedt and H. Rodriguez-Martinez, unpublished). The selective binding of spermatozoa to the epithelium (Suarez, 1998) may account for a reduction in sperm motility, as determined in vitro (Suarez et al., 1991) and, in species other than the pig, in situ (Smith, 1998).

\section{Involvement of the oviduct in sperm capacitation}

Several excellent reviews have been published on sperm capacitation, which is the predominant preparatory event for fertilization (Yanagimachi, 1994; Harrison, 1996; De Lamirande et al., 1997). Sperm capacitation can be defined as a gradual, multi-step process that modifies the architecture of the intrinsically stable sperm plasma membrane (lipid order to disorder and protein relocation), including the removal of epididymal and seminal plasma proteins adsorbed to the sperm plasmalemma during ejaculation, thereby modifying its function in a varying number of cells within a given sperm population. Generally, capacitation includes the removal of membrane cholesterol and the reaction to specific signals by GAGs and glycoproteins, $\mathrm{pH}$ and $\mathrm{Ca}^{2+}$-mediated mechanisms that occur during the sequential passage of spermatozoa in the female genital tract. Bicarbonate is a key effector molecule that elicits the earliest change that accompanies capacitation in pigs, this being the lipid disorder that initiates destabilization of the plasma membrane (Harrison, 1996, 1997; Harrison et al., 1996; Gadella and Harrison, 2000). These membrane modifications precede specific changes in $\mathrm{Ca}^{2+}$ movement, motility patterns, such as hyperactivation, and exocytosis of the acrosome contents during the acrosome reaction, the latter of which is normally elicited upon contact with the zona pellucida (Berger et al., 1989). 
Sperm capacitation in the pig oviduct, assessed with fertilization as the endpoint, is thought to require 5-6 h after mating, but it could take $<2 \mathrm{~h}$ if semen deposition is performed at about the time of ovulation (Hunter, 1990). Data for IVF of pigs indicate that the time required for capacitation of boar spermatozoa in vitro may be $<4 \mathrm{~h}$ (Lynham and Harrison, 1998) and may be even shorter if cryopreserved spermatozoa are used. Surgical insemination of boar spermatozoa into the caudal isthmus and rostral ampulla during periovulation indicated that the caudal isthmus stimulated capacitation (observed as rates of fertilization) more readily than did the ampulla (Hunter et al., 1998).

As boar spermatozoa inseminated at the onset of oestrus may be arrested and maintained viable in the sperm reservoir for $\geqslant 36 \mathrm{~h}$, the role of modulator of sperm capacitation has been ascribed to the reservoir. Capacitation can be modulated by either the intraluminal fluid or by contact between the apical membrane and the isthmic epithelial cells, or both (Suarez et al., 1991; Smith, 1998). Oviductal fluid can reduce polyspermy in pig IVF, possibly owing to the inhibition of the acrosome reaction in the fertilization medium (Kim et al., 1997). The oviductal fluid regulates the rate of capacitation in vivo, this being slower before ovulation than after ovulation (see Rodriguez-Martinez et al., 1998). In addition, epithelial attachment delays rates of sperm capacitation in the reservoir (for review see Rodriguez-Martinez, 2000). Attachment prevents passive $\mathrm{Ca}^{2+}$ inflow to the spermatozoon, maintaining a low intracellular $\mathrm{Ca}^{2+}$ concentration (Dobrinski et al., 1997). A reduction in the metabolism of spermatozoa and a delay in the occurrence of the membrane destabilization that results in capacitation (Harrison et al., 1993) may extend the viability and fertilizing capacity of the stored spermatozoa. As most evidence has been collected in vitro (Harrison, 1996; Fazeli et al., 1999), it remains to be determined more precisely how and when capacitation occurs in vivo.

With this intention, the status of sperm viability and capacitation was studied recently in spermatozoa retrieved by post-mortem flushing from the oviducts of nine sows removed at pre-, peri- or post-ovulation during standing oestrus. The sows (parity 2-5) were observed twice a day for spontaneous oestrus in the presence of fertile boars and were mated with one of two boars at $12 \mathrm{~h}$ after the observed onset of oestrus. The ovaries were explored periodically for preovulatory follicles by transrectal ultrasonography, as described by Mburu et al. (1995). The animals were killed at well-defined stages during oestrus, namely preovulation (at least $8 \mathrm{~h}$ before expected ovulation), periovulation (when follicles started to become less visible or were no longer visible with ultrasonography) or after ovulation (approximately $8 \mathrm{~h}$ after spontaneous ovulation). The oviducts were retrieved and kept at approximately $39^{\circ} \mathrm{C}$ during transportation to a nearby laboratory. The sperm reservoir segments (the uterotubal junction-adjacent lower isthmus) and the ampullary-isthmic junction were flushed with either modified Brackett-Oliphant medium (Brackett and Oliphant, 1975) without $\mathrm{NaHCO}_{3}, \mathrm{BSA}$ or caffeine $(300 \mathrm{mOsmol} \mathrm{I}-1$; control medium or $\mathrm{mBO}$ ), or with $\mathrm{mBO}$ with bicarbonate- $\mathrm{Ca}^{2+}-\mathrm{BSA}$ infused with $5 \% \mathrm{CO}_{2}, \mathrm{pH} 7.4,300 \mathrm{mOsmol}$ $\mathrm{I}^{-1}$ (treatment medium or $\mathrm{mBO}+$ ) at $39^{\circ} \mathrm{C}$ to retrieve the spermatozoa in the $2 \mathrm{~cm}$ segments. The flushings were assessed quickly for the presence of spermatozoa (see Fig. $4 \mathrm{c}$ for a representative SEM view) and examined thereafter at various intervals within 60 min of incubation at $39^{\circ} \mathrm{C}$ for determination of capacitation status. The analyses included flow cytometry (on a FacsStar Plus flow cytometer equipped with standard optics; Becton Dickinson Immunochemistry Systems, San José, CA) of samples loaded with the membrane impermeable, fluorescent lipophilic dye merocyanine-540 $\left(2.7 \mu \mathrm{mol} \mathrm{I}^{-1}\right.$; Molecular Probes Inc., Eugene, OR) and the membrane impermeable nucleic stain Yo-Pro-1 (25 nmol ${ }^{-1}$; Molecular Probes Inc.) (Harrison et al., 1996), as well as incubation with chlortetracycline (Wang et al., 1995). The rationale behind the use of merocyanine-540 was an attempt to recognize the degree of membrane lipid disorder, which is considered to be an early event in 


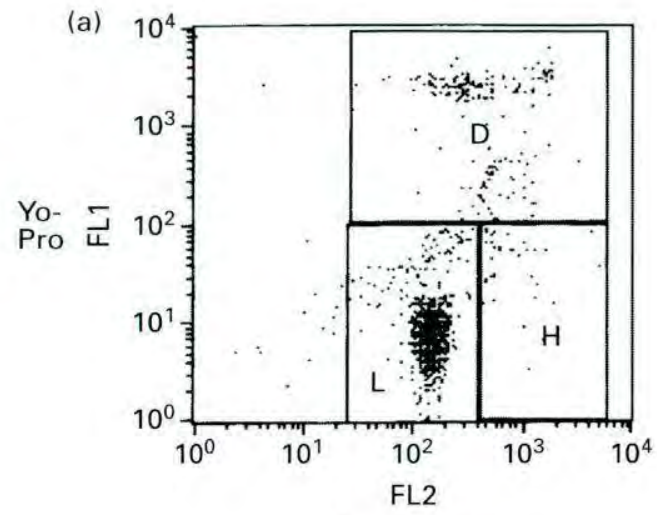

Merocyanine

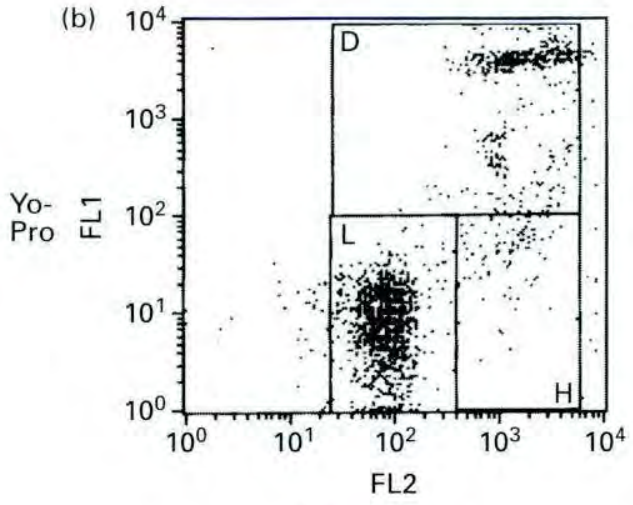

Merocyanine

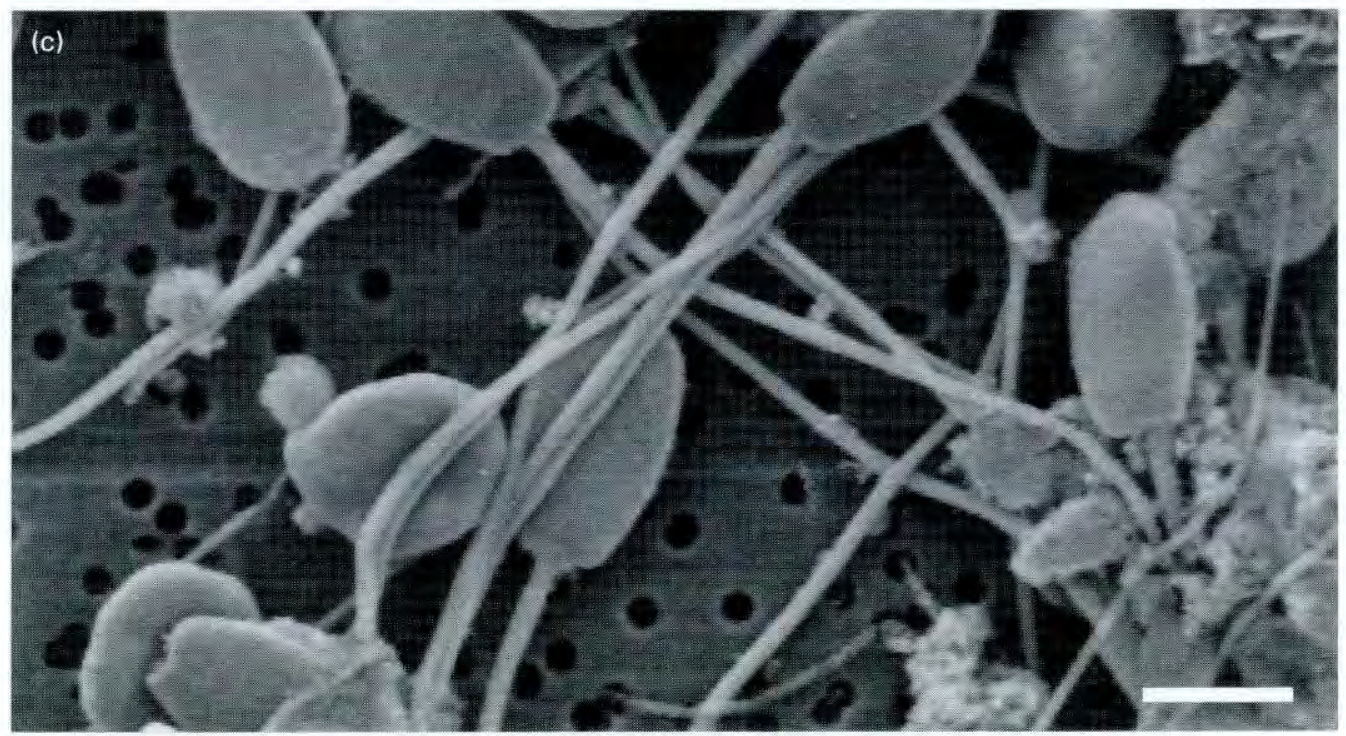

Fig. 4. Flow cytometric dot-plots of boar sperm populations retrieved from the pig sperm reservoir during preovulatory oestrus $(\mathrm{a}, \mathrm{b})$ and incubated in $\mathrm{mBO}+$ containing $2.7 \mu \mathrm{mol}$ merocyanine-540 $\mathrm{I}^{-1}$, $25 \mathrm{nmol}$ Yo-Pro-1 $\mathrm{I}^{-1}, 0.5 \mathrm{mg}$ polyvinylalcohol (PVA) $\mathrm{ml}^{-1}$ and $0.5 \mathrm{mg}$ polyvinylpyrrolidone (PVP) $\mathrm{ml}^{-1}$ for (a) 0 and (b) $30 \mathrm{~min}$. Cells within quadrant ' $\mathrm{L}$ ' are slightly fluorescent with merocyanine-540 (alive, uncapacitated spermatozoa), cells in quadrant ' $\mathrm{H}$ ' are highly fluorescent with merocyanine-540 (capacitated spermatozoa) and cells in quadrant 'D' are fluorescent with Yo-Pro-1 (dead cells). (c) Scanning electron micrograph of representative spermatozoa retrieved by flushing. Scale bar represents $5 \mu \mathrm{m}$.

capacitation (Harrison, 1997; Gadella and Harrison, 2000). Thus, it was hoped that two different subpopulations of live cells, either slightly (low degree of disorder) or highly (high degree of disorder) fluorescent, would be detected on the basis of a mean $10^{4}$ events measured per sample (range from $3 \times 10^{3}$ to $9 \times 10^{4}$ events; Fig. 4 a,b). In addition, it was hoped that different patterns of chlortetracycline fluorescence $\left(\mathrm{a} \mathrm{Ca}^{2+}\right.$ membrane-related event, at 100-200 spermatozoa per sample: uncapacitated (F pattern), capacitated (B pattern) and acrosome-reacted cells) could be distinguished with a light microscope equipped with 


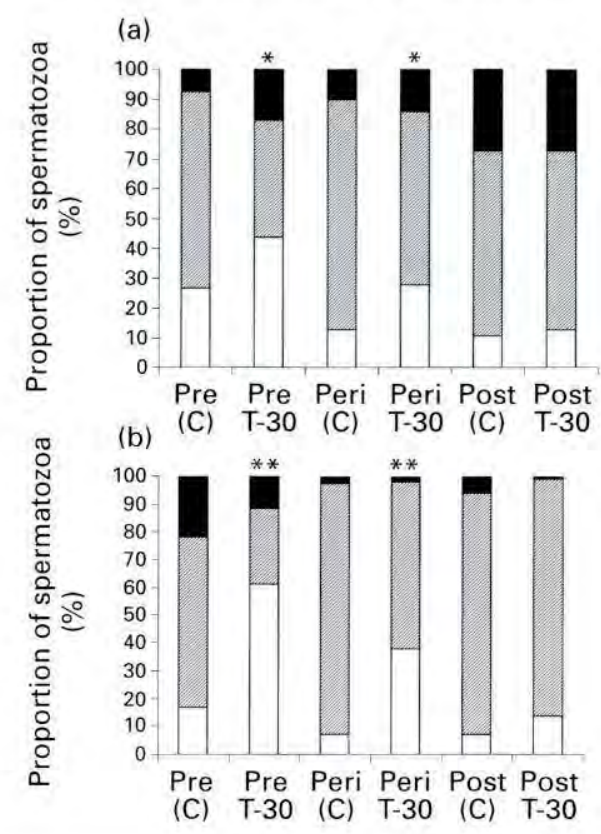

Fig. 5. Subpopulations of boar spermatozoa flushed from the (a) sperm reservoir and (b) ampullary-isthmic junction of sows at preovulatory ( $-8 \mathrm{~h}$; Pre), periovulatory ( $\pm 4 \mathrm{~h}$; Peri) and postovulatory ( $+8 \mathrm{~h}$; Post) stages of standing oestrus. Spermatozoa were flushed with $\mathrm{mBO}$ medium (noncapacitating control; C), loaded with merocyanine-540 and Yo-Pro-1, and examined by flow cytometry. Spermatozoa were incubated thereafter with capacitating medium ( $\mathrm{mBO}+$; containing bicarbonate- $\left.\mathrm{Ca}^{2+}-\mathrm{BSA}\right)$ at $39^{\circ} \mathrm{C}$ for $30 \mathrm{~min}(\mathrm{~T}-30)$ and the flow cytometric analyses were repeated. The histograms depict the relative percentages of cells with high merocyanine540 fluorescence ( $\square$ : capacitated), low merocyanine- 540 fluorescence

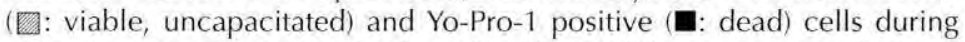
control and treatment incubations. Significant differences were observed between control and treated spermatozoa retrieved from pre- and periovulatory oviducts. ${ }^{*} P<0.05$ and ${ }^{* *} P<0.01$.

epifluorescence optics. As a control procedure, semen collected from the same boars and extended in Beltsville thawing solution (BTS) at a concentration of $1 \times 10^{6}$ spermatozoa ml-1, $^{-1}$ was separated by centrifugation through two layers of $40 \%(\mathrm{v} / \mathrm{v})$ and $80 \%(\mathrm{v} / \mathrm{v})$ Percoll-saline at $300 \mathrm{~g}$ for $15 \mathrm{~min}$ and the cleaned spermatozoa were subjected to incubation in $\mathrm{mBO}$ or $\mathrm{mBO}+$ for up to $300 \mathrm{~min}$. Capacitation status was examined at 5 and $30 \mathrm{~min}$ by flow cytometry (merocyanine-540) and at 15 and $300 \mathrm{~min}$ by chlortetracycline, as described above.

Flow cytometry studies of the viable (Yo-Pro-1 negative) spermatozoa retrieved from the sperm reservoir or the ampullary-isthmic junction (Fig. 5a,b) using control medium (control $\mathrm{mBO})$ indicated that the largest subpopulation of spermatozoa (60-90\%) was uncapacitated, which accounted for the low fluorescence of merocyanine-540. Only minor variations (not significant) in this subpopulation were observed between the different stages of standing oestrus (pre-, peri- or post-ovulation), as the subpopulation of capacitated spermatozoa ranged from $6 \%$ to $37 \%$ of the spermatozoa (Fig. $5 \mathrm{a}, \mathrm{b}$ ). Incubation for $30 \mathrm{~min}$ in the 
capacitation-inducing medium (treatment incubation in $\mathrm{mBO}+$ ) increased the subpopulation of capacitated spermatozoa from the sperm reservoir markedly $(P<0.05)$, but particularly $(P<0.01)$ from the ampullary-isthmic junction at pre- and periovulation (Fig. 5a,b), indicating that spermatozoa might have been uncapacitated but were still viable and responsive to the addition of the effector or inducer bicarbonate (Suzuki et al., 1994). Postovulatory spermatozoa were not affected significantly. In addition, the percentage of dead cells (Yo-Pro- 1 positive cells) often increased with long-term incubation in mBO-capacitating medium compared with controls (Fig. 5a), a reaction that is probably effected by the bicarbonate in the medium, leading to extreme destabilization of the plasmalemma (Harrison et al., 1993). The percentages of cell death were lower in the ampullary-isthmic junction (Fig. 5b) than in the sperm reservoir (Fig. 5a) at the peri- and postovulatory stages, but not at the preovulatory stage. Merocyanine-540 staining showed that there were changes in membrane fluidity in the spermatozoa before the chlortetracycline 'B pattern' was visible, after incubation in uncapacitating medium or capacitating medium, with the chlortetracycline 'B pattern' increasing significantly $(P<0.05)$ after only 60 min of incubation (data not shown). This finding indicates that capacitation follows a long sequence of events (Harrison, 1997) and that each technique shows a particular sequence. The analyses of the ejaculated spermatozoa from the same boars showed clearly that control $\mathrm{mBO}$ was not capacitating but that exposure to $\mathrm{mBO}+$ induced significant $(P<0.05)$ capacitation changes (seven-fold) after only $15 \mathrm{~min}$ of incubation (data not shown). The chlortetracycline analyses were less clear in that changes were observed after $15 \mathrm{~min}$ but similar proportions were also observed after 300 min (data not shown).

Although the above results are from a restricted number of experiments and although tubal flushing does not allow selective retrieval of spermatozoa eventually bound or unbound to the tubal epithelium, the fact that only a small percentage $(<30 \%)$ of spermatozoa showed early signs of capacitation (lipid disorder observed as an increase in merocyanine staining) indicates that capacitation in vivo might not occur in all spermatozoa at once, as is the case with IVF. Hence, these results support the suggestion by Harrison et al. (1993) that "...capacitation might represent a transient stage of (in)stability rather than a discrete physiological state: spermatozoa achieve fertilization only if they happen to be in this transient stage at the time of encountering the egg...". Therefore, these preliminary results would also be in line with previous findings (Rodriguez-Martinez et al., 1998) supporting the suggestion by Smith (1998) that the preovulatory strategy of the isthmus is to delay capacitation rather than to promote it. However, several matters remain to be determined, such as whether the intraluminal secretion delays capacitation or whether this is done by the sperm-epithelium attachment, or alternatively, whether there is synergy between them. Fazeli et al. (1999) used tubal explants from prepubertal pigs to answer this question and concluded that only uncapacitated spermatozoa bind to the oviductal epithelium, whereas capacitated spermatozoa have a reduced binding capacity. In the light of our preliminary results this finding would imply that the flushing was able to retrieve many intact, bound spermatozoa (quite unlikely, in our experience) as well as unbound spermatozoa, which are usually found with disrupted membranes (Mburu et al., 1997). As the percentage of capacitated spermatozoa was low at first reading with merocyanine-540 and even lower with chlortetracycline, the question arises of how many spermatozoa are actually bound or unbound in vivo. Perhaps it is not only the binding that is of importance, but also the medium in which the tubal spermatozoa are found.

In an attempt to answer this question, the effects of exogenous oestrous isthmic oviductal fluid, collected via chronic indwelling catheters, hyaluronan and homologous seminal plasma on the capacitation status of aliquots of flushings of tubal spermatozoa from the sperm 
reservoir were studied. Boar spermatozoa flushed from the sperm reservoir as described above were exposed to capacitating medium ( $\mathrm{mBO}+$ ) without (control) or with the addition of $20 \%$ $(\mathrm{v} / \mathrm{v})$ homologous isthmic oviductal fluid (collected from sows between late pro-oestrus and metoestrus using chronic indwelling catheters, as described above), $500 \mu \mathrm{g}$ hyaluronan $\mathrm{ml}^{-1}$ (Hyonate; Bayer, Gothenburg) or $10 \%(\mathrm{v} / \mathrm{v})$ homologous pooled seminal plasma from the same boars. After 5 and $30 \mathrm{~min}$ of incubation at $39^{\circ} \mathrm{C}$, samples were loaded with merocyanine-540 or Yo-Pro- 1 dye and analysed by flow cytometry. The relative changes in sperm subpopulations after incubation revealed that the percentages of capacitated spermatozoa (high merocyanine-540 fluorescence) in samples incubated with homologous isthmic oviductal fluid and hyaluronan were two- to four-fold lower $(P<0.05)$ than in controls $(\mathrm{mBO}+)$ at pre- and periovulatory periods after either 5 or $30 \mathrm{~min}$ of incubation, whereas no clear effect of seminal plasma was observed (data not shown). The number of dead cells was higher $(P<0.05)$ in all preparations than in controls, particularly when spermatozoa were exposed to seminal plasma $(P<0.01)$. The percentages of viable, uncapacitated cells remained unchanged during the $30 \mathrm{~min}$ of incubation in samples exposed to oviductal fluid and hyaluronan. However, after ovulation, no overall differences were noticed between controls and samples incubated with oviductal fluid, hyaluronan or seminal plasma. In comparison, oviductal fluid, hyaluronan and seminal plasma partially depressed the induction of capacitation in Percoll-cleaned freshly ejaculated spermatozoa, by incubation in bicarbonate-BSA-rich medium ( $\mathrm{mBO}+$, flow cytometry), although they did not reach control values and had a large percentage of destabilized (dead) cells. However, chlortetracycline did not provide the same temporal results as did flow cytometry measurements.

Exposure of frozen-thawed boar spermatozoa to $500 \mu \mathrm{g}$ hyaluronan $\mathrm{ml}^{-1}$ in vitro, under conditions of pig IVF, decreases the incidence of polyspermy (Suzuki et al., 2000), as well as inducing dose-related sperm capacitation (measured with chlortetracycline), without eliciting acrosome exocytosis (K. Suzuki, A. Asano, B. Eriksson, K. Niwa, H. Shimizu, T. Nagai and H. Rodriguez-Martinez, unpublished), thereby indicating that hyaluronan has a protective role relating to sperm viability for this category of preserved spermatozoa. In the above experiments, although there was a decrease in the percentage of capacitated cells compared with controls in samples exposed to oviductal fluid and hyaluronan, the percentages of viable, uncapacitated cells retrieved from the sperm reservoir were higher or remained unchanged during incubation. This finding indicates that the sperm reservoir fluid helps to maintain sperm viability, perhaps because of its hyaluronan content. However, more studies are required before conclusions can be drawn. Exposure to seminal plasma at similar concentrations has been demonstrated to maintain frozen-thawed boar spermatozoa in the chlortetracycline 'F pattern' and, furthermore, to counteract oocyte penetration by liquid-stored or frozen-thawed spermatozoa (K. Suzuki, A. Asano, B. Eriksson, K. Niwa, H. Shimizu, T. Nagai and H. Rodriguez-Martinez, unpublished). Such a preventive effect on capacitation was not observed in the tubal spermatozoa when chlortetracycline was used and it was also not clear with flow cytometry (merocyanine-540) analysis of ejaculated spermatozoa in the preliminary experiments reported in this review.

\section{Progression of spermatozoa from the sperm reservoir}

There is a sequential progression of boar spermatozoa from the reservoir towards the ampullary-isthmic junction in relation to ovulation (Mburu et al., 1996, 1997). This progression may be the consequence of several processes that are not necessarily mutually exclusive. Boar spermatozoa may achieve hyperactivation in the reservoir, detach from the epithelium and progress towards the ampullary-isthmic junction. To our knowledge, there is 
no evidence that this occurs in pigs in vivo. Furthermore, the lifespan of pig spermatozoa might be too short (Yanagimachi, 1994) to enable them to cover the distance to the ampullary-isthmic junction individually. However, a constant release and progression of spermatozoa that are not necessarily capacitated may occur as a consequence of a synergistic, concerted action of oedema resumption, changes in viscosity (perhaps in relation to hyaluronidase activity), an increased flow of tubal fluid and co-ordinated myosalpinx contractility towards the ampullary-isthmic junction (see Rodriguez-Martinez et al., 1998). Once they have left the sperm reservoir, spermatozoa may, probably on an individual basis and as an expression of the heterogeneity of the ejaculate, be readily capacitated, perhaps at very different rates, by the high concentrations of bicarbonate $\left(>30 \mathrm{mmol} \mathrm{I}^{-1}\right)$ present in the ampullary-isthmic junction (E. Ekstedt and $H$. Rodriguez-Martinez, unpublished). These concentrations are similar to those that facilitated capacitation in the spermatozoa flushed from the ampullary-isthmic junction (Fig. 5b) at pre- or periovulation (but not after ovulation, this being at $>8 \mathrm{~h}$ ). Similar concentrations are used for ejaculated spermatozoa in pig IVF (Suzuki et al., 1994). Periovulatory fluid collected from the pig ampullary-isthmic junction increased the number of ejaculated spermatozoa depicting hyperactivation-like motility in vitro ('circling motility'; Nichol et al., 1997), thus supporting this hypothesis. Hormonal changes such as the action of preovulatory progesterone (Hunter, 1995) may underlie some of these mechanisms, rather than acting as a single signal triggering massive sperm progression.

No matter which mechanism is responsible, a regulation of the capacitation rate in the reservoir would ensure that appropriate numbers of fertilizing spermatozoa are present when ovulation occurs, thereby maximizing the likelihood of fertilization when sperm deposition is performed well ahead of ovulation, a common situation in pigs mated naturally. As capacitated spermatozoa have a relatively short lifespan (Yanagimachi, 1994), the likelihood of fertilization once they have left the reservoir and its stabilizing milieu depends largely on the number and location of viable spermatozoa along the oviduct mid-region, which is a fairly low number in vivo, according to Mburu et al. (1996). Considering the short period of ovulation (about $3 \mathrm{~h}$ ) and the rapid transport of ova in pigs $(<1 \mathrm{~h}$ ), there is a requirement for spermatozoa at different stages of capacitation to cover the area in which the ova may appear to provide the best chances for successful, timed fertilization. This requirement may also account for the large sperm population characteristic of pig ejaculates.

\section{Role of the oviduct in oocyte pick-up, transport and oocyte development}

Like the spermatozoon, the oocyte must reach full maturity before it is ready for fertilization and the inherent completion of meiosis and triggering of zygote cleavage. The preovulatory gonadotrophin surge signals release from meiotic arrest and completion of meiosis I, such that pig oocytes reach the metaphase II stage just before ovulation. At ovulation, oocytes are picked up by the cilia-covered fimbriae and guided through the infundibulum and ampulla (Oxenreider and Day, 1965; Alanko, 1974). The in vivo collection of oocytes in pigs is complex and is aided by the frictional large ovarian bursa and the contraction of selected muscles located in the mesosalpinx, such as the ligamentum infundibulo-cornuale. In vivo, the oocyte remains arrested at metaphase II until activated by fusion with the fertilizing spermatozoon (Alanko, 1974). In pigs, the rate of ampullar transport towards the ampullary-isthmic junction is fairly rapid (30-45 $\mathrm{min}$ ) and the newly ovulated oocytes in their cumulus vestment aggregate within a 'cumulus plug' (Hancock, 1961), as shown (Fig. 6a-c). How oocyte pick-up and transport are regulated and elicited is still not clear as the ova are transported against a flow of tubal fluid. It is possible that the concerted action of an adhesive interaction between cumulus cells and the extracellular matrix they synthesize (Talbot et al., 
1999) (mainly hyaluronan) and ciliary beating, as well as myosalpingeal peristalsis (Rodriguez-Martinez et al., 1982), are major agents. Eventually, final denudation of cumulus cells occurs, probably in relation to the presence of sperm hyaluronidase close to the plug, released by those spermatozoa that have broken acrosomes and are often observed in large numbers in the central lumen of the upper isthmus (Mburu et al., 1997), exposing the zona pellucida to the tubal fluid (Fig. 6e). Molecules present in the oviductal lumen are involved in the development of the oocytes. Some of these are simple macromolecules, such as hyaluronan, which is present in the fluid during the entire period of tubal functionality, that is, until metoestrus (Tienthai et al., 2000). Hyaluronan can support the development of pig oocytes fertilized in vitro up to the blastocyst stage (Kano et al., 1998). Specific tubal proteins called 'porcine oviductal secretory proteins (POSPs)', produced de novo under oestrogendependence and occurring during late pro-oestrus, oestrus and metoestrus, become associated with the oocyte (Gandolfi, 1995; Buhi et al., 1997, 2000). Ultrastructural studies have demonstrated that POSPs traverse the zona pellucida and associate with the oolemma and the plasma membrane of pig blastomeres (Buhi et al., 1997, 2000). The role of such proteins is not fully understood but it is related to trophism, the enhancement of sperm-zona pellucida binding, extracellular matrix formation and prevention of premature hatching (Buhi et al., 1997).

\section{Impairment of fertilization in pigs}

Capacitation represents a conflict between the maintenance of sperm viability and destabilization that, if expressed fully before the sperm-oocyte encounter, renders the spermatozoon infertile and ultimately causes cell death (Harrison, 1996). If insemination takes place $>24 \mathrm{~h}$ before ovulation, fertilization rates decrease, owing to low numbers of fertile spermatozoa present at the site of fertilization. Although sperm ageing has a clear effect on fertilization and conceptus development (Shaver and Martin-De Leon, 1977), oocyte ageing has been viewed as a major cause of fertilization failure in pigs (Kim et al., 1996a). At sperm penetration, oocytes prevent the multiple entry of spermatozoa at the zona pellucida by the release of cortical granules, which cause a zona pellucida reaction. It is thought that postovulatory ageing of mammalian oocytes before sperm penetration may lead to polyspermy, owing to a non-functional cortical reaction mechanism, caused in turn by a delayed and incomplete exocytosis of the cortical granules (Hunter, 1994b). Although increasing progesterone concentrations after ovulation may be a causative factor, an increase in the number of spermatozoa ascending a more patent isthmus from the reservoir is the main cause of polyspermy (Hunter, 1994b), which is the major obstacle for optimal IVF in pigs. However, recent evidence indicates that the abnormally high incidence of polyspermic penetration of pig oocytes in vitro is probably not due to delayed or incomplete cortical granule exocytosis but to a delayed zona pellucida reaction, allowing simultaneous sperm penetration (Wang et al., 1998). The surface morphology of the zona pellucida and the zona pellucida reactions at sperm penetration differs between in vitro-matured oocytes derived from offal and ovulated oocytes, as detected by SEM (Funahashi et al., 2000). This observation can perhaps be explained in relation to the presence of additional macromolecules (Hedrick et al., 1987) that make the outer surface of the zona pellucida smoother (Fig. 6d). The oestrogen-dependent oviductal glycoproteins secreted into the oviductal lumen are of particular interest (Brown and Cheng, 1986; Buhi et al., 1997). These substances in the intraluminal oviductal fluid may be required for the final maturation of the zona pellucida in in vitro-matured pig oocytes, for example by providing an increased resistance to pronase digestion (Kim et al., 1996b) or a reduction of polyspermy (Vatzias and Hagen, 1999) when in 

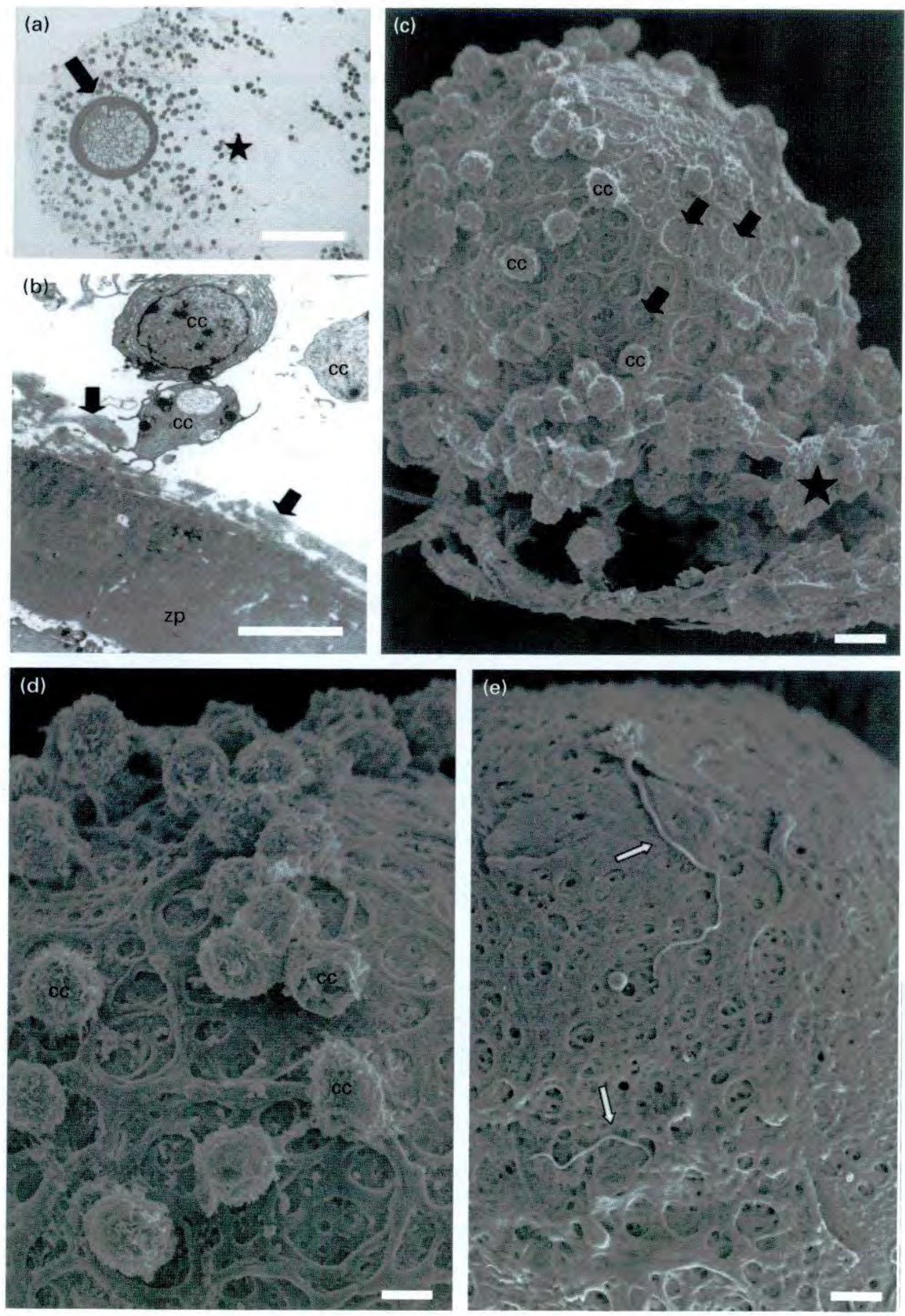

Fig. 6. Oocytes retrieved from pig ampullary-isthmic junction during periovulatory oestrus. (a) Low magnification photomicrograph of a partial view of an oocyte plug. Arrow: oocyte adjoined to the other oocytes (eight in total, not all included in the picture) by a cloud of extracellular inert material (star) among isolated cumulus cells. Spermatozoa do not seem to have reached the cloud yet. (b) Transmission electron micrograph of the oocyte in (a). Note the aspect of the zona pellucida (zp) with a spongy appearance (arrows) 
vitro-matured oocytes are exposed to oviductal fluid. Funahashi et al. (2001) explored the ultrastructure of pig zonae pellucidae by transmission electron microscopy and confirmed that there are differences in the zona pellucida and the zona pellucida reaction at sperm penetration between in vivo- and in vitro-fertilized oocytes, with clear differences in network organization between the inner and outer zona pellucida. During final maturation, the zona pellucida of in vivo-ovulated oocytes may add oviductal glycoproteins, which allow the complete change in conformation of the inner zona pellucida that characterizes the zona pellucida reaction to occur.

\section{Conclusion}

The preliminary results presented in this review using merocyanine-540 dye and flow cytometry to monitor the early stages of sperm capacitation (lipid disorder in the sperm plasma membrane) showed that the largest subpopulation, $60-90 \%$, of viable spermatozoa retrieved from the sperm reservoir and the ampullary-isthmic junction of mated sows at preand periovulation was uncapacitated. Incubation in a capacitation-inducing medium (mBO with bicarbonate-BSA) for $<30 \mathrm{~min}$ induced capacitation readily, more markedly in samples from the ampullary-isthmic junction than in those from the uterotubal junction. These results indicate that these uncapacitated spermatozoa were responsive to the addition of the effector bicarbonate at concentrations similar to those recorded in the periovulatory ampullaryisthmic junction in vivo. The addition of preovulatory isthmic oviductal fluid and hyaluronan under a similar incubation regimen appeared to retain the viability of tubal spermatozoa. This finding indicates that sperm reservoir fluid, perhaps because of its hyaluronan content, might be responsible for delaying sperm capacitation in the uterotubal junction-isthmus. In this study, evidence is provided for the need for a diverse response to capacitation by the sperm population in the oviduct in vivo that would ensure full rates of fertilization, indicating that caution is required when extrapolating results obtained solely from in vitro studies. On the basis of ultrastructural studies of the zona pellucida of in vivo- and in vitro-developed oocytes it is evident that the oviduct is also responsible for the final maturation of the zona pellucida by secreting specific glycoproteins that result in proper zona pellucida reaction after sperm penetration, and can explain in part the abnormal occurrence of polyspermy in pig IVF.

The skilled technical support of $M$. Ekwall and $\AA$. Jansson is acknowledged. Funding has been provided by FORMAS, formerly the Swedish Council for Forestry and Agricultural Research (SJFR), the Swedish Farmers' Foundation for Agricultural Research (SL.F), and the Swedish Foundation for International Cooperation in Research and Higher Education (STINT), through the SLU-Japan Programme on Reproductive Biotechnology, Sweden.

and cumulus cells (cc) still associated with the zona pellucida. (c) Scanning electron micrograph (SEM) of an oocyte as in (a) yet almost completely covered by cumulus cells (cc) and extracellular material (star). Note the rather wide fenestrations in the zona pellucida (arrows). (d) Higher magnification of (c) showing the outer surface of the zona pellucida with wide fenestrations left by the cumulus cell outgrowths. (e) SEM of another oocyte denuded of cumulus cells, depicting two sperm tails (arrows) that have penetrated the zona pellucida partially. Note the aspect of the outer zona pellucida and compare it with the amorphous cross-sectioned oocyte in (c). Scale bars represent (a) $100 \mu \mathrm{m}$, (b,d,e) $5 \mu \mathrm{m}$ and (c) $10 \mu \mathrm{m}$. 


\section{References}

Alanko M (1974) Fertilization and Early Development of Ova in Al-Cilts, with Special Reference to the Role of Tubal Sperm Concentration: A Clinical and Experimental Study PhD Thesis, University of Helsinki

Berger T, Turner DO, Meizel S and Heidrick IL (1989) The zona pellucida-induced acrosome reaction in boar sperm Biology of Reproduction 40 525-530

Brackett BG and Oliphant C (1975) Capacitation of rabbit spermatozoa in vitro. Biology of Reproduction $\mathbf{1 2}$ 260-274.

Brown CR and Cheng WKT (1986) Changes in composition of the porcine zona pellucida during development of the oocyte to the 2- 10 4-cell embryo Journal of Embryology and Experimental Morphology 92 183-191

Buhi WC, Alvarez IM and Kouba AJ (1997) Oviductal regulation of fertilization and early embryonic development Journal of Reproduction and Fertility Supplement 52 285-300

Buhi WC, Alvarez IM and Kouba AJ (2000) Secreted proteins of the oviduct Cells, Tissues, Organs 166 165-179

De Lamirande E, Leclerc P and Gagnon C (1997) Capacitation as a regulatory event that primes spermatozoa for the acrosome reaction and fertilization Molecular Human Reproduction 3 175-194

Dobrinski I, Smith T, Suarez SS and Ball BA (1997) Membrane contact with oviductal epithelium modulates the intracellular calcium concentration of equine spermatozoa in vitro. Biology of Reproduction $56861-869$

Fazeli A, Duncan AE, Watson PF and Holt WV (1999) Sperm-oviduct interaction: induction of capacitation and preferential binding of uncapacitated spermatozoa to oviductal epithelial cells in porcine species Biology of Reproduction 60 879-886

Funahashi H, Ekwall H and Rodriguez-Martinez H (2000) The zona reaction in porcine oocytes fertilized in vivo and in vitro as seen with scanning electron microscopy Biology of Reproduction 63 1437-1442

Funahashi $H$, Ekwall $H$, Kikuchi $K$ and Rodriguez-Martinez H (2001) Transmission electron microscopy studies of the zona reaction in pig oocytes fertilized in vivo and in vitro. Reproduction 122 443-452

Gadella BM and Harrison RAP (2000) The capacitating agent bicarbonate induces protein kinase A-dependent changes in phospholipid transbilayer behaviour in the sperm plasma membrane Development 127 2407-2420

Gandolfi F (1995) Functions of proteins secreted by oviduct epithelial cells Microscopy Research Techniques 32 $1-12$

Hancock JL (1961) Fertilization in the pig fournal of Reproduction and Fertility 2 307-331

Harrison RAP (1996) Capacitation mechanisms, and the role of capacitation as seen in eutherian mammals Reproduction, Fertility and Development 8 581-594

Harrison RAP (1997) Sperm plasma membrane characteristics and boar semen fertility Journal of Reproduction and Fertility Supplement 52 195-2 11

Harrison RAP, Mairet B and Miller NGA (1993) Flow cytometric studies of bicarbonate-mediated $\mathrm{Ca}^{2+}$ inilux in boar sperm populations Molecular Reproduction and Development 35 197-208

Harrison RAP, Ashworth PJC and Miller NGA (1996) Bicarbonate/ $\mathrm{CO}_{2}$, an effector of capacitation, induces a rapid and reversible change in the lipid architecture of boar sperm plasma membranes Molecular Reproduction and Development 45 378-391

Hedrick JL, Wardrip NJ and Berger T (1987) Differences in the macromolecular composition of the zona pellucida isolated from pig oocytes, eggs, and zygotes Journal of Experimental Zoology 241 257-262

Hunter RHF (1990) Fertilization of pig eggs in vivo and in vitro. Journal of Reproduction and Fertility Supplement 40 211-226

Munter RHF (1994a) Modulation of gamete and embryonic microenvironments by oviduct glycoproteins Molecular Reproduction and Development 39 176-181

Hunter RHF (1994b) Causes for failure of fertilization in domestic species. In Embryonic Mortality in Domestic Species pp 1-22 Eds MT Zavy and RD Geisert. CRC Press, Boca Raton

Hunter RHF (1995) Ovarian endocrine control of sperm progression in the Fallopian tubes Oxford Reviews of Reproductive Biology 17 85-124

Hunter RHF, Huang WT and Holtz W (1998) Regional influences of the Fallopian tubes on the rate of boar sperm capacitation in surgically inseminated gilts Journal of Reproduction and Fertility 114 17-23

Johansson M, Tienthai P and Rodriguez-Martinez H (2000) Histochemistry and ultrastructure of the intraluminal mucus in the sperm reservoir of the pig oviduct fournal of Reproduction and Development 46 183-192

Kano K, Miyano T and Kato A (1998) Effects of glycosaminoglycans on the development of in vitro. matured and fertilized porcine oocytes to the blastocyst stage in vitro. Biology of Reproduction 58 1226-1232

Kavanaugh JF, Grippo AA and Killian GJ (1992) Cannulation of the bovine ampullary and isthmic oviduct Journal of Investigative Surgery 5 11-17

Kim NH, Moon SJ, Prather RS and Day BN (1996a) Cytoskeletal alteration in aged porcine oocytes and parthenogenesis Molecular Reproduction and Development 43 513-518

Kim NH, Funahashi H, Abeydeera LR, Moon SJ, Prather RS and Day BN (1996b) Effects of oviductal fluid on sperm penetration and cortical granule exocytosis during in vitro fertilization of porcine oocytes fournal of Reproduction and Ferility 107 79-86

Kim NH, Day BN, Lim JG, Lee HT and Chung KS (1997) Effects of oviductal fluid and heparin on fertility and characteristics of porcine spermatozoa Zygote 5 61-65

Leese HJ, Tay J, Reischl I and Downing SJ (2001) Formation of Fallopian tubal fluid: role of a neglected epithelium Reproduction 121 339-346

Lynham IA and Harrison RAP (1998) Use of stored eggs to assess boar sperm fertilizing functions in vitro. Biology of Reproduction 58 539-550

Mburu JN, Einarsson S, Dalin AM and Rodriguez-Martinez H (1995) Ovulation as determined by transrectal 
ultrasonography in multiparous sows: relationships with oestrous symptoms and hormonal profiles journal of Veterinary Medicine A 42 285-292

Mburu JN, Einarsson S, Lundeheim $\mathbf{N}$ and RodriguezMartinez H (1996) Distribution, number and membrane integrity of spermatozoa in the pig oviduct in relation to spontaneous ovulation Animal Reproduction Science 45 109-121

Mburu JN, Rodriguez-Martinez $\mathbf{H}$ and Einarsson S (1997) Changes in sperm ultrastructure and localisation in the porcine oviduct around ovulation Animal Reproduction Science 41 137-148

Nichol R, Hunter RHF, Gardner DK, Leese HJ and Cooke GM (1992) Concentrations of energy substrates in oviductal fluid and blood plasma during the periovulatory period Journal of Reproduction and Fertility 96 699-707

Nichol R, Hunter RHF, De Lamirande E, Gagnon C and Cooke GM (1997) Motility of spermatozoa in hydrosalpingeal and follicular fluid of pigs journal of Reproduction and Fertility 110 79-86

Nichol R, Hunter RHF, Gardner DK, Partridge R, Leese HJ and Cooke GM (1998) Concentrations of energy substrates in oviductal filuid in unilaterally ovariectomized pigs Research in Veterinary Science 65 263-264

Oxenreider SL and Day BN (1965) Transport and cleavage of ova in swine fournal of Animal Science 24 413-417

Rodriguez-Martinez $\mathbf{H}$ (2000) The oviduct of the pig: do intraluminal glycosaminoglycans play a role in tubal function? In Boar Semen Preservation N pp 153-163 Eds LA Johnson and HD Guthrie. Allen Press Inc., Lawrence

Rodriguez-Martinez H, Einarsson S and Larsson B (1982) Spontaneous motility of the oviduct in the anaesthetized pig Journal of Reproduction and Fertility 66 615-624

Rodriguez-Martinez $\mathrm{H}$, Nicander L, Viring S, Einarsson S and Larsson K (1990) Ultrastructure of the uterotubal junction in preovulatory pigs Anatomia Histologia Embryologia 19 16-36

Rodriguez-Martinez $H$, Larsson B, Pertoft $H$ and Kjellén $L$ (1998) GACs and spermatozoon competence in vivo and in vitro. In Cametes: Development and Function pp 239-274 Eds A Lauria, F Gandolfi, G Enne and L. Gianaroli. Serono Symposium, Serono

Shaver EL and Martin-De Leon DC (1977) Effects of aging of sperm in the female and male reproductive tracts before fertilization on the chromosome complement of the blastocyst Aging Gametes 16 151-165

Smith $\Pi$ (1998) The modulation of sperm function by the oviductal epithelium Biology of Reproduction $\mathbf{5 8}$ 1102-1104

Suarez SS (1998) The oviductal sperm reservoir in mammals Biology of Reproduction 58 1105-1107

Suarez, SS, Redferm K, Raynor P, Martin F and Philips DM (1991) Attachment of boar sperm to mucosal explants of oviduct in vitro: role in formation of a sperm reservoir Biology of Reproduction 44 998-1004

Suzuki K, Ebihara M, Nagai T, Clarke NGE and Harrison RAP (1994) Importance of bicarbonate/ $\mathrm{CO}_{2}$ for fertilization of pig aocytes in vitro and synergism with caffeine Reproduction, Fertility and Development 6 221-227

Suzuki K, Eriksson B, Shimizu H, Nagai T and RodriguezMartinez (2000) Effecl of hyaluronan on monospermic penetration of porcine oocytes fertilized in vitro. international journal of Andrology 23 13-21

Talbot P, Geiske C and Knoll M (1999) Oocyte pickup by the mammalian oviduct Molecular Biology of the Cell to 5-8

Tienthai P, Kjellén L, Pertoft H, Suzuki K and RodriguezMartinez $H(2000)$ Localisation and quantitation of hyaluronan and sulphated glycosaminoglycans in the tissues and intraluminal fluid of the pig oviduct Reproduction, Fertility and Development 12 173-182

Vatzias G and Hagen DR (1999) Effects of porcine follicular fluid and oviduct-conditioned media on maturation and fertilization of porcine oocyles in vitro. Biology of Reproduction $6042-48$

Wang WH, Abeydeera LR, Fraser LR and Niwa K (1995) Functional analysis using chlortetracycline íluorescence and in vitro fertilization of frozen-thawed ejaculated boar spermatozoa incubated in a protein-free chemically defined medium Journal of Reproduction and Fertility 104 305-313

Wang WH, Abeydeera LR, Prather RS and Day BN (1998) Morphologic comparison of ovulated and in vitromatured porcine oocytes, with particular reference to polyspermy after in vitro fertilization Molecular Reproduction and Development 49 308-316

Yanagimachi R (1994) Mammalian fertilization. In The Physiology of Reproduction pp 189-317 Eds E Knobil and JD Neill. Raven Press, New York 